\title{
Cluster Source Deposition Based Hierarchical Nanoporous Ag Film Deposited Substrate for Surface Enhanced Raman Scattering
}

\author{
Sungho Yun ${ }^{1,2}$, Junyeop Lee', Jaemoon Yang ${ }^{3}$, Dongin Lee ${ }^{4}$, Bonghwan Kim,", and Chanseob Cho ${ }^{1, *}$ \\ ${ }^{1}$ School of Electronics Engineering, Kyungpook National University \\ Daegu 41566, Korea \\ ${ }^{2}$ Kwang-Lim Precision, Inc. \\ Daegu 43013, Korea \\ ${ }^{3}$ Yonsei University \\ Seoul 03722, Korea \\ ${ }^{4}$ Department of Information and Communication Engineering, Yeungnam University \\ Gyeogbuk 38541, Korea \\ ${ }^{5}$ School of Electronics and Electrical Engineering, Dauge Catholic University \\ Gyeongbuk 38430, Korea \\ bhkim@cu.ac.kr*; chocs@knu.ac.kr*corresponding author
}

\section{Extended Abstract}

By mounting a cluster source on a conventional sputtering system, a new sputtering system is devised for the deposition of nanoporous metal thin films at room temperature. First, the diameter of the nozzle is fixed while the pressure inside the cluster source is kept at several hundreds of mTorr. By adjusting the length of the cluster source, nanoporous films of different thickness can be formed at room temperature. The Raman response characteristics according to the process conditions are then analyzed, and the applicability of the sputtered thin film as a substrate for surface-enhanced Raman scattering (SERS) is examined [1-6].

The Raman intensity increased with increasing thickness and showed a tendency to be saturated approximately $4.3 \times$ $10^{4} \mathrm{cps}$ in $2 \mu \mathrm{m}$. As the process pressure increased, the gap between the metal clusters increased and the porosity also increased to $71-82 \%$. When the length of the condensation region was varied between $135 \mathrm{~mm}$ and $214 \mathrm{~mm}$, the EF of the Raman had a value between $3.97 \times 10^{6}$ and $4.44 \times 10^{6}$. When the partial pressure ratio of He is $7.5 \%$, EF is $4.06 \times 10^{6}$. It increases to $4.4 \times 10^{6}$ when the partial pressure of He is increased to $17.6 \%$. However, it decreases when the partial pressure of He increases further. As for power, we observed that EF is $3.54 \times 10^{6}$ when the power is $120 \mathrm{~W}$. It increases to $4.44 \times 10^{6}$ when the power is $140 \mathrm{~W}$. However, it decreases when the power is $160 \mathrm{~W}$. Therefore, the proposed system takes advantage of an existing sputtering process, and it is possible to form a nanoporous metal film with thickness of several micrometers or more that can be used as a Raman substrate [7-12].

\section{Acknowledgements}

This research was supported by the Basic Science Research Program through the National Research Foundation of Korea (NRF) funded by the Ministry of Education (NRF-2017R1D1A3B03034258); the Basic Science Research Program through the National Research Foundation of Korea (NRF) funded by the Ministry of Education (No. NRF2016R1D1A3A03919627); and the Ministry of Science, ICT and Future Planning (MSIP), Korea, under the Information Technology Research Center (ITRC) support program (IITP-2017-2016-0-00313) supervised by the Institute for Information \& communications Technology Promotion (IITP).

\section{References}

[1] D. K. Lim, K. S. Jeon, J. H. Hwang, H. Kim, S. Kwon, Y. D. Suh, and J. M. Nam, Nat. Nanotechnol., vol. 6, p. 452 2011.

[2] Z. Wang, L. Tang, L. H. Tan, J. Li, and Y. Lu, Angew. Chem. Int. Ed Engl., vol. 51, p. 9078, 2012.

[3] A. Otto, I. Mrozek, H. Grabhorn, W. Akemann, Journal of Physics: Condensed Matter, vol. 4, no. 5, p. $1143,1992$. 
[4] S. A. Maier, Plasmonics: Fundamentals and Applications, Springer, Atlanta, 2007.

[5] J. Stropp, G. Trachta, G. Brehm, and S. Schneider, Journal of Raman Spectroscopy, vol. 34, no. 1, p. $26,2003$.

[6] J. F. Li, Y. F. Huang, Y. Ding, Z. L. Yang, S. B. Li, X. S. Zhou, F. R Fan, W. Zhang, et al., Nature, vol. 464, p. 392 2010.

[7] W. C. Lin, L. S. Liao, Y. H. Chen, H. C. Chang, D. P. Tsai, and H. P. Chiang, Plasmonics, vol. 6, no. 2, p. $201,2011$.

[8] E. D. Diebold, N. H. Mack, S. K. Doorn, and E. Mazur, Langmuir, vol. 25, no. 3, p. 1790, 2009.

[9] J. Fontana, J. Livenere, F. J. Bezares, J. D.Caldwell, R. Rendell, and B. R. Ratna, Applied Physics Letters, vol. 102, no. 20, p. 201606, 2013.

[10] K. C. Bantz, A. F. Meyer, N. J. Wittenberg, H. Imb, Ö. Kurtuluş, S. H. Lee, N. C. Lindquist, S.-H. Oh, and C. L. Haynes, Phys Chem Chem Phys., vol. 13, no. 24, p. 11551, 2011.

[11] H. Qiu, M. Wang, S. Jiang, L. Zhang, Z. Yang, L. Li, J. Li, M. Cao, and J. Huang, Sensors and Actuators B, vol. 249, no. 437, 2017.

[12] C. Yang, Y. Qin, X. Zhu, M. Yin, D. Li, X. Chen, and Y. Song, J. Alloys and Compounds, vol. 632, p. 634, 2015. 